\title{
EARLY DIAGNOSIS OF THE UROFACIAL SYNDROME IS ESSENTIAL TO PREVENT IRREVERSIBLE RENAL FAILURE
}

\author{
FRANCISCO A. NICANOR, ANTHONY COOK, JOAO L PIPPI-SALLE
}

\author{
Division of Urology, Hospital for Sick Children, University of Toronto, Toronto, Ontario, Canada
}

\begin{abstract}
Introduction: The urofacial or Ochoa syndrome is a rare disease characterized by the presence of functional obstructive uropathy associated with peculiar facial features when patients attempt to smile or laugh. Unfortunately, many of these patients remain without proper diagnosis or adequate treatment due to lack of recognition of the disease. This can ultimately result in upper tract deterioration and eventual renal failure. We present our experience with this rare syndrome.

Materials and Methods: We identified 3 patients who presented initially with acute renal failure, urinary tract infection (UTI) and severe dysfunctional elimination. All patients were thoroughly evaluated, including screening for spinal cord anomalies, and were subsequently diagnosed with urofacial syndrome.

Results: At the outset, the two older patients (aged 4 and 9 years) presented with the typical facial features when attempting to smile or laugh. One patient in the newborn period presented with urinary and fecal retention and septicemia and, to our knowledge, represents the youngest case of urofacial syndrome reported so far. All patients were evaluated with ultrasonography, renal scan, voiding cystourethrogram (VCUG) and urodynamics. Findings included hydronephrosis and a thickwalled, trabeculated bladder with poor compliance and detrusor hypereflexia respectively in each patient. All were subsequently treated with clean intermittent catheterization (CIC), antibiotic prophylaxis and anticholinergic therapy. One patient required appendicovesicostomy for CIC due to discomfort secondary to a sensate urethra.

Conclusions: Our series demonstrates that early recognition of this rare syndrome is necessary to adequately treat and prevent upper tract deterioration in these unique individuals. Although the urofacial is difficult to diagnose in infants, cognizance must be maintained in order to prevent severe subsequent sequalae.
\end{abstract}

Key words: bladder neurogenic; spastic neurogenic bladder; renal failure; syndrome; facies Int Braz J Urol. 2005; 31: 477-81

\section{INTRODUCTION}

In the early 1960s, Bernardo Ochoa followed a group of children with the characteristic symptomatology of neurogenic bladder (recurrent urinary infection, incontinence, constipation, dysuria, frequency, enuresis, bladder trabeculation and vesicoureteral reflux) with no apparent neurological or obstructive abnormality. Furthermore, each patient showed a particular facial expression, i.e., grimacing when they attempted to smile or laugh (1). This unusual disorder was initially considered a local observation, but today over 100 patients have been reported in literature. 
The urofacial or Ochoa syndrome is a rare disease that occurs in both sexes and is more frequent when the parents are closely related. It has been determined to be inherited by an autosomal recessive pattern (2). A potential gene has been mapped to chromosome 10q23-q24 (1). Patients with Ochoa syndrome present with functional obstructive uropathy, residual urine, and hydroureteronephrosis. Symptoms are variable and may consist of urinary tract infection (UTI), urgency, frequency, incontinence, polyuria, and polydipsia. Many present with elimination dysfunction (60\% constipation and 33\% encopresis) (3), and have a characteristic pathognomonic inversion of the facial expression when they attempt to smile or laugh. Interestingly, patients demonstrate emotionally congruent facial expressions when sad or crying.

The laughing and crying centers are located in upper pons of the midbrain close to the micturition center and it has been speculated that this is the possible pathophysiological explanation for this dysfunctional facial characteristic (4).

\section{MATERIALS AND METHODS}

We present our experience with three patients with urofacial syndrome. All of them presented initially with acute renal failure, UTI and severe dysfunctional elimination. Follow-up ranged from 2-9 years (average 5 years). All patients underwent voiding cystourethrogram (VCUG), abdominal ultrasound, urodynamic studies and dimercaptosuccinic acid (DMSA) scintigraphy. Spinal magnetic resonance imaging (MRI) was performed in 2 cases.

\section{RESULTS}

Case 1: A 4-day-old Caucasian female presented acute urinary and fecal retention. Her symptoms initially spontaneously resolved, however at 48 days of age, she returned to hospital with urinary retention and septicemia. Peritoneal dialysis was started for acute renal failure. Ultrasound showed bilateral hydroureteronephrosis. Voiding cystourethrogram (VCUG) showed bladder trabeculation, grade $\mathrm{V}$ bilateral reflux and urinary retention. Clean intermit- tent catheterization (CIC) was initiated, but upon familial noncompliance a vesicostomy was performed. Urodynamics showed a poorly compliant, hyperactive bladder, high voiding pressures and detrusorsphincter dyssynergia. Treatment with anticholinergic (propantheline bromide $3 \mathrm{mg} / \mathrm{kg} /$ day) resulted in improved bladder capacity and resolution of reflux. Vesicostomy was closed and CIC started at 3 years of age. A trial was attempted with an alpha-blocker (doxazosin) at a dose of $2 \mathrm{mg} /$ day for 6 months without any improvement whatsoever. Nuclear medicine with dimercaptosuccinic acid (DMSA) showed normal kidneys bilaterally. She is presently 9 years old, continues on CIC every 4 hours and remains infection free. Familial pedigree demonstrates no history of Ochoa syndrome. Figure-1 demonstrates the typical facial grimaces associated with the syndrome.

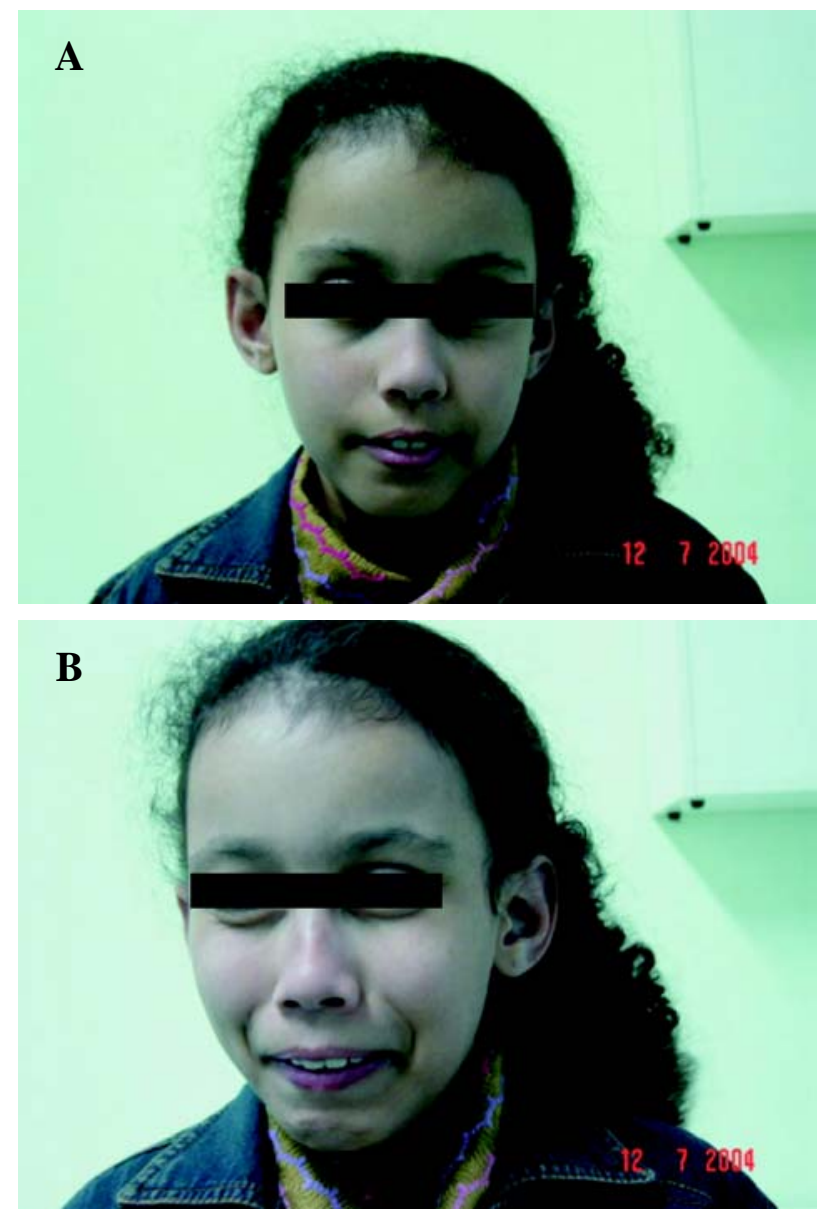

Figure 1 - Typical facial grimaces associated with Ochoa syndrome. 
Case 2: A4-year-old Caucasian male, a product of conception between two first cousins, was initially referred to the nephrology clinic for evaluation of hydronephrosis, polyuria and polydipsia. At that time, 14 hour fasting revealed normal urea, creatinine and serum sodium, effectively ruling-out diabetes insipidus. He was concomitantly evaluated by pediatric ophthalmology for non-specific visual disturbances and subsequently underwent a cranial MRI, which was negative for underlying neuro-pathology. He then went to a local emergency department following an episode of gross painless hematuria. Evaluation at that time included an abdominopelvic CT scan that revealed moderate right hydronephrosis. His gross hematuria spontaneously resolved and he was discharged with the diagnosis of acute viral cystitis. Thereafter, he was referred for urologic evaluation and was found to have a long history of irritative and obstructive voiding symptoms. At this time he had elevated creatinine and urea. Physical examination, including the elucidation of a smile, demonstrated the typical urofacial grimacing (Figure-2). The parents revealed that two aunts had similar smile findings but no urinary retention at any time. Evaluation, including renal ultrasonography and VCUG, demonstrated moderate bilateral hydroureteronephrosis and a thickwalled, trabeculated bladder with no evidence of vesico-ureteral reflux (VUR) or posterior urethral valve (PUV) respectively (Figure-3). An MRI of the spine was normal. Urodynamic evaluation demonstrated a poorly compliant, hyperactive bladder with elevated filling pressures. Bilateral renal scarring was noted upon DMSA renal scan (Figure-4). He was initially treated with anticholinergic therapy and CIC, but refused catheterizations from the outset due to a sensate urethra, and an appendicovesicostomy was performed to ease catheterization. Follow-up over the past 2 years has demonstrated normalization of serum creatinine and excellent compliance with respect to CIC every 2 hours plus ongoing high doses of anticholinergic therapy, which resulted in significant improvement of the hydroureteronephrosis.

Case 3: A 9-year-old Caucasian female was referred to the urologic clinic for evaluation of daytime and nocturnal urinary incontinence, urgency and recurrent urinary tract infection. Physical examination
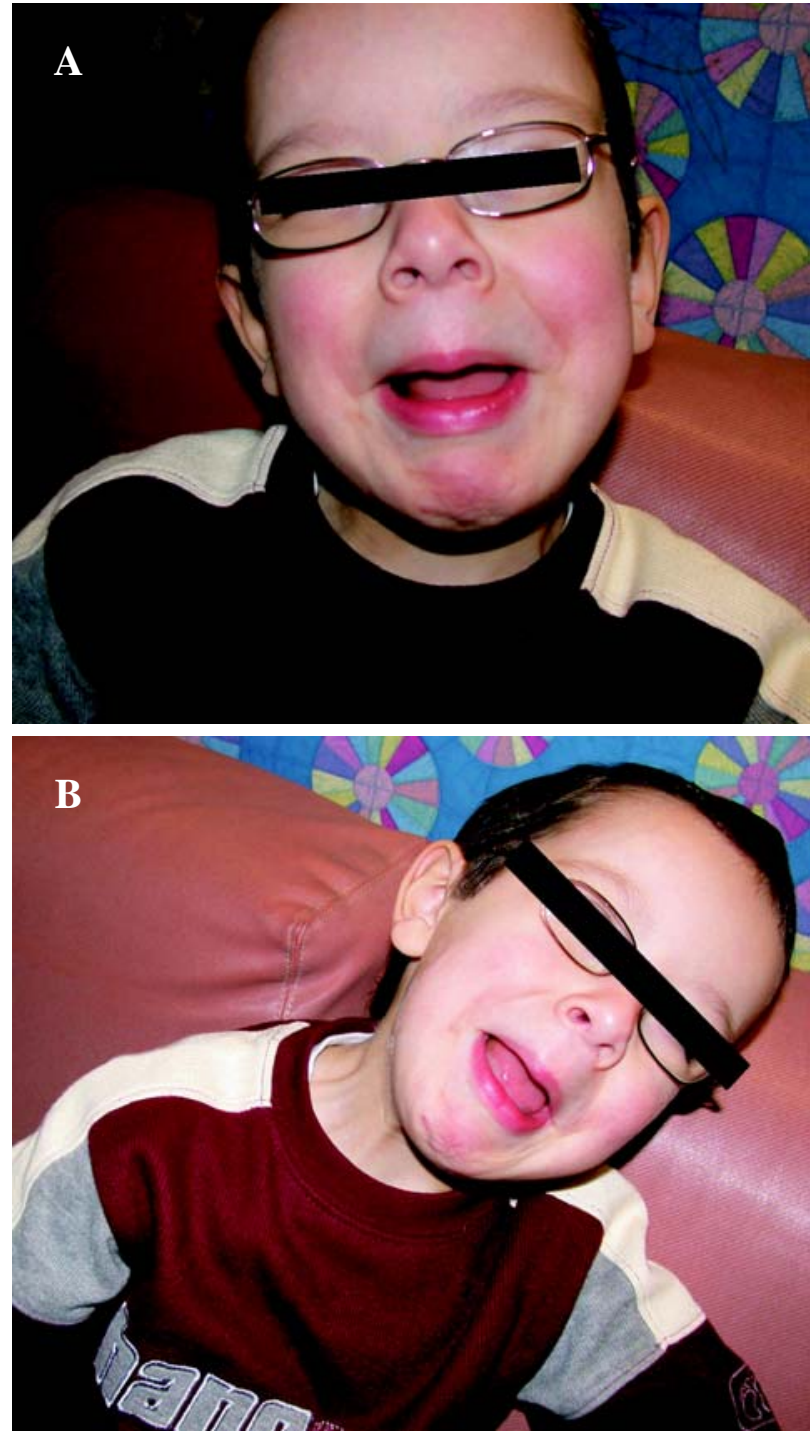

Figure 2 - Typical facial grimaces associated with Ochoa syndrome.

revealed the classical finding of Urofacial Syndrome, having the typical smile expression (Figure-5). VCUG demonstrated bladder trabeculation and bilateral grade III VUR. Renal scarring was noted bilaterally upon DMSA renal scan. Urodynamics showed a poorly compliant bladder and detrusor hyperactivity. MRI demonstrated a normal spine and conus medularis. The patient was started on progressive doses of oxybutinin (up to $7.5 \mathrm{mg}$ tid) and CIC, which resulted in markedly improved urinary symptoms. Finally, her family history was also negative for Ochoa syndrome. 

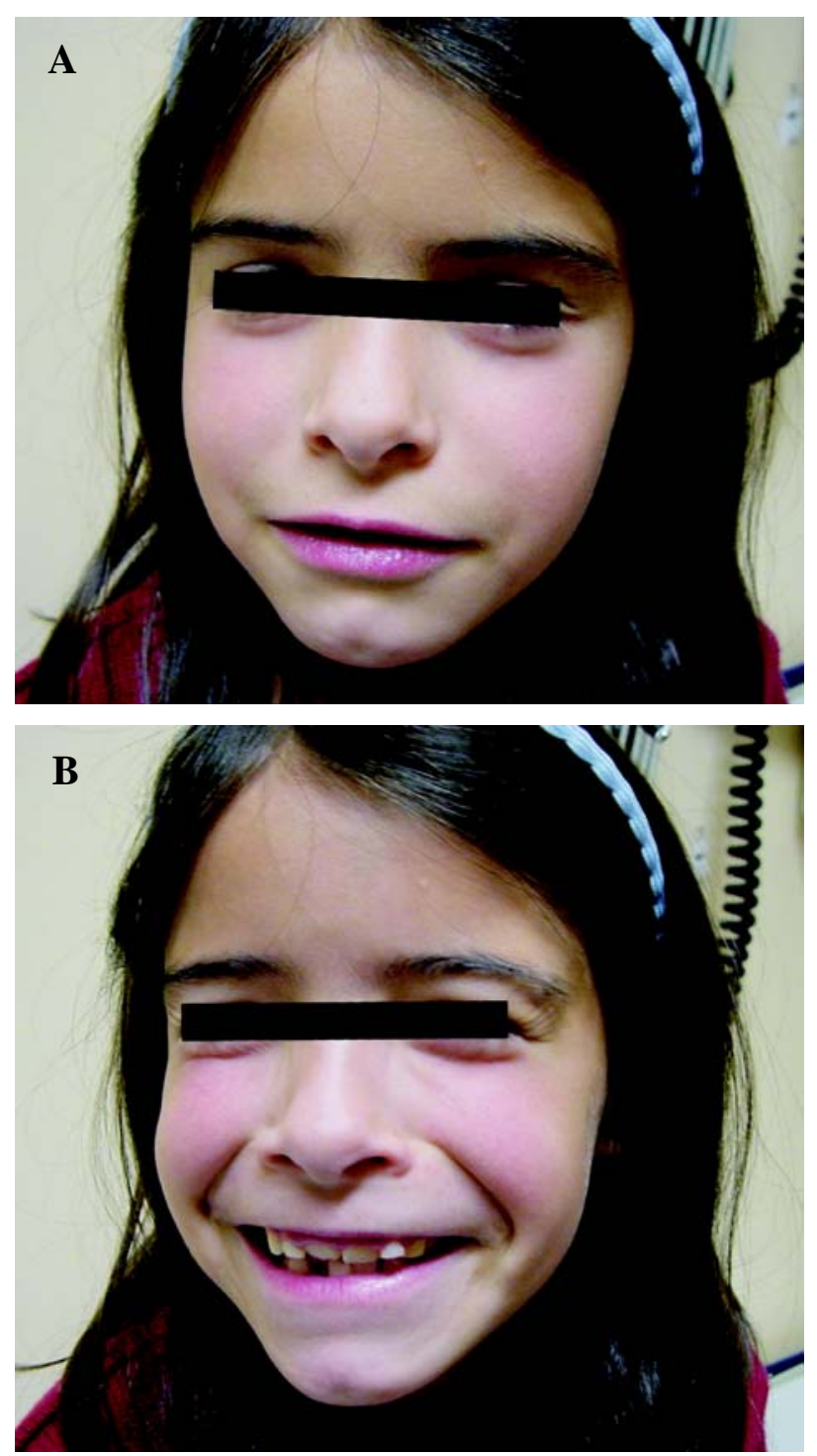

Figure 3 - Typical facial grimaces associated with Ochoa syndrome.

\section{COMMENTS}

Although rare, it is important (and extremely simple) to exclude the urofacial syndrome in patients who present with dysfunctional voiding or dysfunctional elimination. Early diagnosis and treatment may prevent irreversible damage to the urinary tract system.

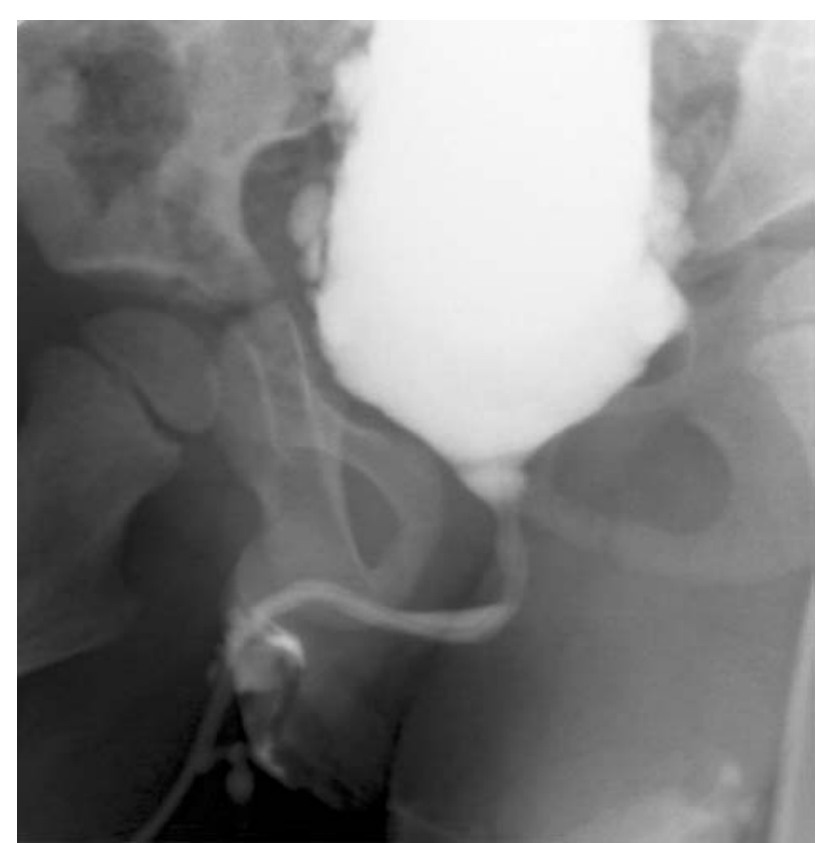

Figure 4 - VCUG: bladder trabeculation and multiple pseudodiverticula.

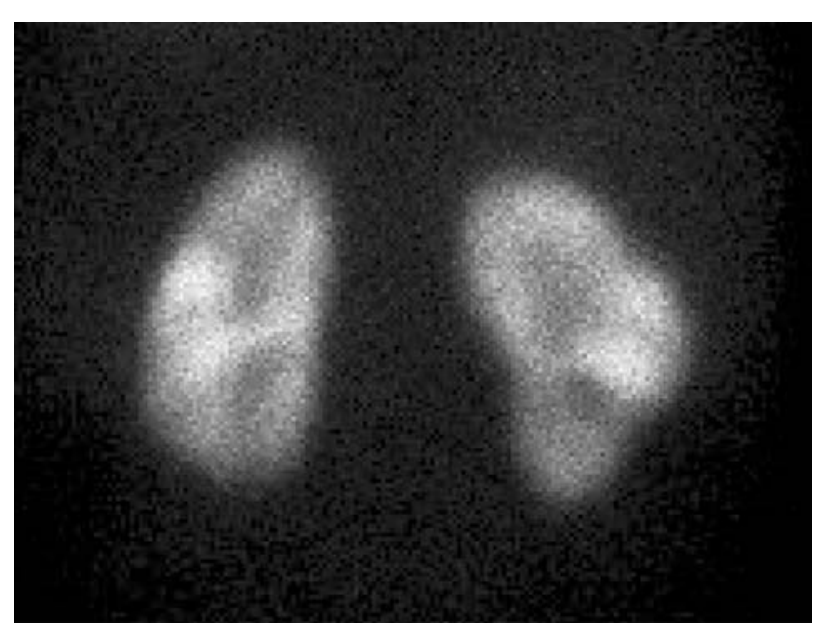

Figure 5-DMSA shows foci of decreased cortical function in both kidneys.

Previously, genetic studies have been carried out in patients with Ochoa syndrome. The observations of normal parents with several affected siblings and parental consanguinity at that time made it clear that inheritance was autosomally recessive, as in our second case $(2,5)$. It is possible that patients with non-neurogenic bladder or Hynman syndrome, subclinical neurological bladder, occult neu- 
ropathic bladder, dysfunctional voiding, or dysfunctional elimination may be affected by the same congenital neurologic disorder at the level of the pontine micturition center. However, those with Ochoa syndrome also appear to have concomitant subclinical lesions in the laughing and crying center (1). As spinal lesion may be a potential underlying disorder, an MRI should be performed to rule out other possible etiologies, such as cord tethering, before reaching a final diagnosis.

The presence of renal scarring should be noted and monitored by DMSA renal scans. Urodynamics are important not only to document abnormalities but also for follow-up and disease prognostication. Initially, detrusor hyperreflexia is associated with sphincter dyssinergia, however, in advanced cases, myogenic detrusor failure may ultimately result (6).

The aim of treatment is the restoration of balanced bladder emptying and the prevention of upper tract deterioration. Drug therapy is guided by urodynamic findings and includes anticholinergics and antibiotic prophylaxis if secondary UTI persist despite regular bladder emptying by CIC. Intermittent catheterization is fundamental and patients with sensate urethras may require continent urinary diversion, such as appendicovesicostomy. Polyuric patients should undergo nocturnal bladder emptying with an indwelling nighttime catheter, as this has been shown to improve overall lower and upper urinary tract function (7).

\section{CONCLUSION}

Although rare, the urofacial syndrome is an important, well-recognized entity, which, if not treated adequately, may lead to significant morbidity and even mortality from progressive renal deterioration. One must maintain cognizance and screen for this disorder in patients with a history of severe dysfunctional voiding by greeting each patient with a smile at the time of initial consultation.

\section{REFERENCES}

1. Ochoa B: Can a congenital dysfunctional bladder be diagnosed from a smile? The Ochoa syndrome updated. Pediatr Nephrol. 2004; 19: 6-12.

2. Ochoa B, Gorlin RJ: Urofacial (Ochoa) syndrome. Am J Med Genet. 1987; 27: 661-7.

3. Feng WC, Churchill BM: Dysfunctional elimination syndrome in children without obvious spinal cord diseases. Pediatr Clin North Am. 2001; 48: 1489-504.

4. Ochoa B: The urofacial (Ochoa) syndrome revisited. J Urol. 1992; 148: 580-3.

5. Elejalde BR: Genetic and diagnostic considerations in three families with abnormalities of facial expression and congenital urinary obstruction: "The Ochoa syndrome". Am J Med Genet. 1979; 3: 97-108.

6. Garcia-Minaur S, Oliver F, Yanez JM, Soriano JR, Quinn F, Reardon W: Three new European cases of urofacial (Ochoa) syndrome. Clin Dysmorphol. 2001; 10: $165-70$.

7. Koff SA, Mutabagani KH, Jayanthi VR: The valve bladder syndrome: pathophysiology and treatment with nocturnal bladder emptying. J Urol. 2002; 167: 291-7.

Received: June 26, 2005

Accepted after revision: July 25, 2005

\author{
Correspondence address: \\ Dr. Francisco A. M. Nicanor \\ 80577 Elm Street M5G 1H4 \\ Toronto, Ontario, Canada \\ E-mail: nicanoramacedo@hotmail.com
}

